\title{
Near-Field Chipless-RFID System with High Data Capacity for Security and Authentication Applications
}

\author{
Cristian Herrojo, Student Member IEEE, Javier Mata-Contreras, Alba Núñez, Ferran Paredes, Member \\ IEEE, Eloi Ramon, and Ferran Martín, Fellow IEEE
}

\begin{abstract}
A high data capacity chipless radiofrequency identification (chipless-RFID) system, useful for security and authentication applications, is presented in this paper. Reading is based on near-field coupling between the tag, a chain of identical split ring resonators (SRRs) printed on a (typically flexible) dielectric substrate (e.g., liquid crystal polymer, plastic, paper, etc.), and the reader. Encoding is achieved by the presence or absence of SRRs at predefined (equidistant) positions in the chain, and tag identification is based on sequential bit reading. Namely, the tag must be longitudinally displaced, at short distance, over the reader, a microstrip line loaded with a SRR and fed by a harmonic signal. By this means, the harmonic signal is amplitude modulated, and the identification (ID) code is contained in the envelope function, which can be obtained by means of an envelope detector. With this system, tag reading requires proximity with the reader, but this is not an issue in many applications within the domain of security and authentication (e.g., secure paper for corporate documents, certificates, etc.). Several circularly-shaped 40-bit encoders (implemented in a commercial microwave substrate), and the corresponding reader, are designed and fabricated as proof-of-concept demonstrators. Strategies for programming the tags and a first proof-of-concept chipless-RFID tag fabricated on plastic substrate through inkjet printing are included in the paper.
\end{abstract}

Index Terms- Split ring resonators (SRRs), microstrip technology, chipless-RFID.

\section{INTRODUCTION}

Chipless radiofrequency identification (chipless-RFID) is an alternative to chipped-RFID systems for the identification, tracking and/or authentication of objects and items, where the silicon integrated circuit (IC) is replaced with a printable passive encoder [1]-[6]. Such encoder contains a unique signature that can be identified by means of a RF (or microwave) interrogation signal in frequency, time or hybrid domains. The main advantage of chiplessRFID over RFID systems based on tags equipped with microchips is the low cost of the encoders, which can be fabricated by means of (additive) printing techniques, such as screen printing, rotogravure, flexography or inkjet, or, obviously, by means of photo-etching. However, size and data capacity (crucial aspects of any identification system) of printable encoders reported to date are not competitive with

This paper is an expanded version of the paper [41], published in IEEEMTT-S International Microwave Symposium.

This work was supported by MINECO-Spain (projects TEC2013-40600R, TEC2016-75650-R and RTC-2014-2550-7), Generalitat de Catalunya (project 2014SGR-157), Institució Catalana de Recerca $i$ Estudis Avançats (who awarded F. Martín), and by FEDER funds. C. Herrojo acknowledges the negligible dimensions and high memory storage capacity of silicon ICs.

Many efforts have been made in recent years to alleviate the previous limitations of chipless-RFID tags. There are two main approaches for data encoding in chipless RFID: time domain reflectometry (TDR) [7]-[16] and spectral signature [1],[2],[17]-[36]. TDR based tags exhibit fast responses as compared to frequency domain tags, but their bit encoding capability is limited since tag ID is generated by the echoes of a pulsed signal (caused by discontinuities or impedance mismatches), and either large delay lines or very narrow pulses are needed to avoid overlapping of the reflected pulses. Therefore, most efforts to increase the data storage capacity have been focused on frequency-domain based tags. In this case, encoders are based on resonant elements tuned at predefined frequencies covering a certain spectral bandwidth. Encoding is achieved by the presence or absence of abrupt spectral features in the amplitude, phase or group delay responses, and tag reading requires a multi-frequency interrogation signal covering the whole spectral bandwidth of interest.

Spectral signature barcodes (as they are usually designated to point out the similarity with optical barcodes) can be of two main types: retransmission based [17],[18] and backscattered based [19],[25] tags. The former consists of a transmission line loaded with resonant elements (or with resonant elements coupled to it) and two cross polarized antennas, one for reception and one for transmission, so that interference between the interrogation signal and the retransmitted encoded signal is minimized. By contrast, in backscattered tags, the resonant elements act as receiving and transmitting antennas and provide the spectral signature through the radar cross section (RCS) peaks. Typically, backscattered chipless tags are more compact since antennas are not required.

In frequency-domain based tags, the number of bits is typically given by the number of resonant elements. Strategies to enhance the number of bits without an increase in the number of resonators (and hence bandwidth and size) include multi-state multi-resonator tags [34]-[36] and hybrid tags [27],[30]-[33]. The former exploits the fact that more than two states can be achieved by a single resonant element.

MINECO for supporting his research activity through the FPI grant BES2014-068164.

C. Herrojo, J. Mata-Contreras, F. Paredes and F. Martín are with GEMMA/CIMITEC, Departament d'Enginyeria Electrònica, Universitat Autònoma de Barcelona, 08193 Bellaterra, Spain. E-mail: Ferran.Martin@uab.es.

A. Núñez and E. Ramon are with Institut de Microelectrònica de Barcelona, IMB-CNM (CSIC), 08193 Bellaterra, Spain. 
Particularly, in retransmission based tags the multi-state functionality can be obtained through the controllability of the attenuation level of notches (amplitude response) achieved by rotation [35],[36]. Hybrid tags are multi-domain tags where more than one domain (e.g. time, frequency, phase, polarization, etc.) are used simultaneously in order to achieve more than one bit of information per resonant element. Examples of hybrid tags include encoders based on frequency position and polarization diversity [31], and encoders where frequency domain is combined with phase deviation [30], among others.

In spite of the recent efforts to increase the number of bits, mainly focused on increasing the spectral efficiency (bits/GHz), the reported chipless RFID tags are far from the data capacity of chipped tags. In [36], it was shown that by sacrificing reading distance, and by using the concept of near field (inductive) coupling between the tag and the reader, an information density per frequency of 16 bits/GHz can be achieved. In such multi-state multi-resonator tags, implemented by S-shaped SRRs (S-SRRs), a resonant particle introduced in [37]-[39] and used in several applications (e.g., sensors [40]), the resonant elements are etched in a different dielectric layer than the transmission line, a coplanar waveguide (CPW) acting as reader (frontend). Tag reading requires proper alignment and contact between the tag and the transmission line (reader), which is not necessarily an issue in certain applications such as security and authentication, as reported in [36]. However, it is difficult in practice to increase the information density per surface and frequency by means of these tags since it is not possible to increase the number of states per resonator beyond four (as in [36]).

In this paper, a different and unconventional approach, first reported in [41], to significantly enhance the number of bits of chipless tags is used for the implementation of 40-bit chipless tags. The approach is based on sequential bit reading, and it is achieved by displacing the tag, a chain of identical resonators, over a transmission line (reader) fed by a harmonic signal. The presence or absence of resonant elements at predefined equidistant positions in the resonator chain modulates the input (carrier) signal, so that the ID code is present in the envelope of the modulated signal. Note that the interrogation signal is simply a harmonic signal. In these new chipless RFID tags the information is given by the presence or absence of resonant elements at predefined positions. Equivalently, the ID code, obtained from the envelope function in time domain, is given by the presence or absence of notches at predefined times. Conceptually, the working principle for tag reading in these chipless RFID tags is similar to the one of the angular velocity sensors reported in [42], [43].

The main innovation of the proposed system comes in the form of a reduced form factor of the tags by cutting out antennas, not using spectral features, and using sequential bit reading through motion of the tag over the reader. As compared to the work in [41], we report here a chipless RFID system where the reader is a microstrip line loaded with a split ring resonator (SRR) [44],[45] and the resonator chain uses the same resonant elements but rotated $180^{\circ}$. The implementation of the active part of the reader in microstrip technology is important for backside isolation. Moreover, vias are not necessary. An important difference as compared to the chipless RFID system proposed in [41] concerns the fact that the SRR-loaded line (reader) reported here is configured as a bandpass filter with a deep notch above the pass band. The harmonic feeding signal is tuned to the frequency of maximum transmission. However, the system is designed in such a way that when a SRR of the tag lies on top of the SRR of the reader, the frequency response experiences a shift and the carrier frequency is strongly attenuated due to the shift in the transmission zero position. With this strategy, the dynamic range, or modulation index, is enhanced, as compared to [41]. We report 40-bit chipless RFID tags, which are circularly shaped for proper reading through a step motor used previously by the authors for angular velocity measurements [42],[43].

The work is organized as follows. The working principle is succinctly summarized in Section II. The layout of the reader and the topology of the resonant elements are presented in Section III. The lumped element equivalent circuit model of the structure and the method to extract the parameters are also included in this section. In section IV, an exhaustive analysis of the proposed system, useful to determine the convenient carrier frequency of the feeding signal, is carried out. The effects of the air gap separation and lateral misalignments are also discussed in this section. The experimental set-up for tag reading, as well as the fabricated tags, a set of four 40-bit tags implemented in the four quadrants of a circularly shaped narrow and flexible dielectric layer, is presented in Section V. The envelopes inferred from tag reading, corresponding to the different chipless tags, and providing the ID codes, are also provided in Section V. Section VI is devoted to a discussion on different strategies for programmable tags based on resonator detuning, and it is demonstrated that by mechanically cutting the resonators along their symmetry plane, detuning, and consequently tag reconfigurability, is achieved. In this section, it is also demonstrated that the proposed chiplessRFID system works by printing the tags on plastic substrates, of interest in many applications including security and authentication. Finally, the main conclusions are highlighted in Section VII.

\section{WORKING PRINCIPLE}

The working principle of the proposed chipless RFID system is based on near-field coupling between the tag, a chain of identical resonant elements (SRRs), and the active part of the reader, a SRR-loaded microstrip line configured as bandpass filter. The SRR of the line is identical to the SRRs of the tags, but rotated $180^{\circ}$. The ID code is inferred sequentially and by proximity; namely, the SRR chain of the tag must be longitudinally (i.e., in the direction of the line axis) displaced above the position of the SRR of the line. The distance between the line SRR and the SRR chain (air gap separation) must be small, in order to favor the coupling between the SRR of the line and those of the chain.

Through tag motion, the frequency response of the SRRloaded line varies substantially provided the coupling between the line and tag SRRs is efficiently modulated. To this end, small distances (air gap) are necessary. Moreover, 


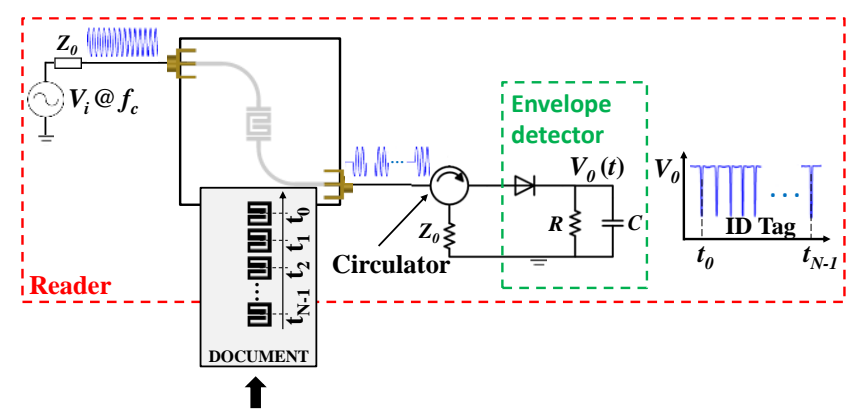

Fig. 1. Sketch of the proposed chipless RFID system, consisting of the tag (set of resonators printed or etched on a substrate) and the reader (within the dashed rectangle), constituted by the harmonic generator, the SRR-loaded transmission line, the circulator, and the envelope detector.

the relative $180^{\circ}$ orientation between the SRR of the line and those of the tag chain is necessary, since such orientation enhances the coupling in the optimum position (i.e., the one with perfectly aligned line and tag SRRs) [46],[47]. By contrast, when the tag chain is located with the intermediate positions between two adjacent SRRs just on top of the center of the line SRR, the coupling is negligible. Tag motion results in shifts in the frequency response which are intimately related to the coupling level for the vertically aligned SRRs. The consequence of these shifts is the modulation of the transmission coefficient of the SRRloaded line with tag motion. If a harmonic (carrier) signal tuned to a certain frequency is injected to the input port of the SRR-loaded line, the signal at the output port is modulated by tag motion, and the ID code is contained in the envelope of the modulated signal. The sketch of the proposed chipless RFID system is depicted in Fig. 1.

In the proposed chipless RFID tags, the logic ' 1 ' and ' 0 ' states are given by the presence or absence of SRRs at predefined and equidistant positions in the chain. To enhance sensitivity, it is convenient to choose the carrier frequency exhibiting the maximum excursion (variation) of the transmission coefficient with tag motion. Through this choice, the modulation index is optimized, and the logic states can be better discerned in a reading operation. This aspect will be discussed in Section IV. Nevertheless, the SRR-loaded microstrip structure acting as bandpass filter provides a large excursion between maximum and minimum transmission, and this represents a clear advantage as compared to the system proposed in [41].

\section{READER AND TAG TOPOLOGIES AND LUMPED ELEMENT EQUIVALENT CIRCUIT MODEL}

The active part of the reader, a microstrip line loaded with a SRR, is depicted in Fig. 2. The SRR is folded in order to reduce its electrical size. As mentioned before, the SRRs of the tag chain are identical, but rotated $180^{\circ}$ (hence the topology is not repeated). In view of Fig. 2, it follows that the structure exhibits a band pass behavior, and filter bandwidth is related to the width of the slot between the resonant element and the access lines, $s_{2}$. The frequency response of the structure of Fig. 2, inferred from full wave electromagnetic simulation (using Keysight Momentum), is depicted in Fig. 3 (the parameters of the Rogers RO3010 substrate, with thickness $h=0.635 \mathrm{~mm}$, dielectric constant $\varepsilon_{r}$ $=10.2$ and loss tangent $\tan \delta=0.0022$, have been

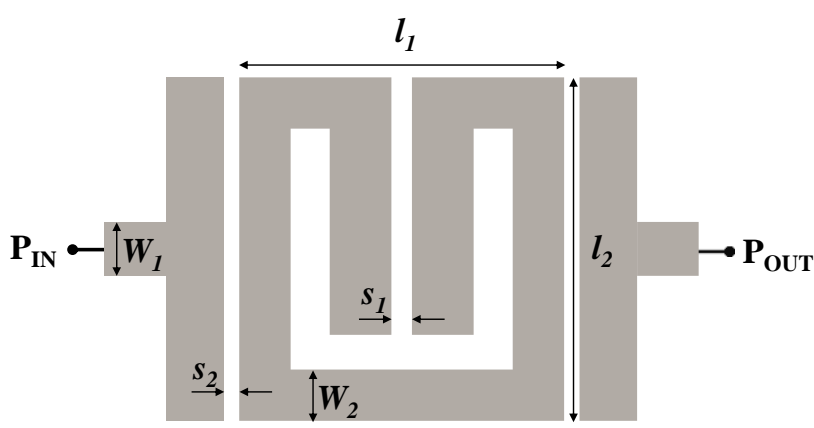

Fig. 2. Topology of the reader. Dimensions are (in mm): $l_{l}=3.16, l_{2}=3.35$, $s_{l}=0.2, s_{2}=0.2, W_{l}=0.56$, and $W_{2}=0.5$. The distance between adjacent SRRs (it they are present) in the tag chain is $0.2 \mathrm{~mm}$.

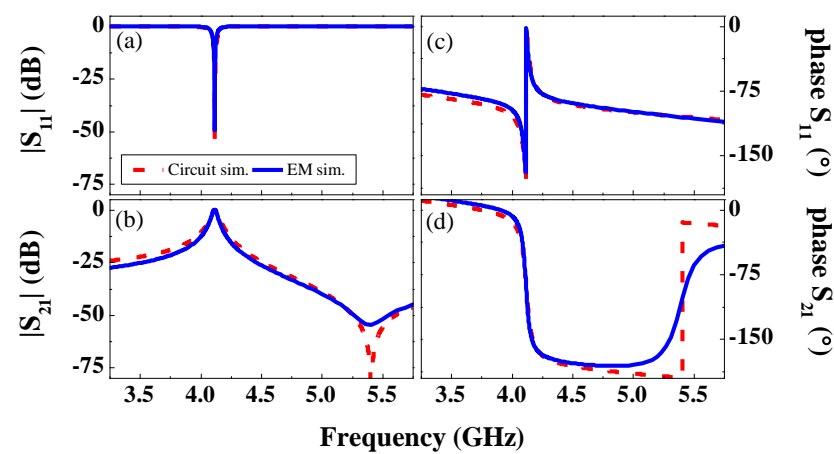

Fig. 3. Frequency response of the structure of Fig. 2 inferred from lossless electromagnetic and circuit simulation. (a) Magnitude of $S_{11}$; (b) magnitude of $S_{21}$; (c) phase of $S_{11}$; (d) phase of $S_{21}$. The extracted parameters of the lumped element equivalent circuit (in reference to Fig. 4) are: $L_{r}=27.1 \mathrm{nH}$, $C_{r}=0.03 \mathrm{pF}, C_{g}=0.05 \mathrm{pF}$ and $C_{p}=0.76 \mathrm{pF}$.

considered). The transmission coefficient exhibits a transmission zero, given by the intrinsic resonance frequency of the SRRs, and a pole (or reflection zero), where all the injected power is transmitted to the output port (neglecting the effect of losses). This response is very useful for our purposes, due to the large insertion loss at the transmission zero frequency (with direct impact on the modulation index of the output signal in a reading operation, as will be later shown).

The structure of Fig. 2 can be described by the lumped element $\pi$-circuit model depicted in Fig. 4. The parallel resonant tank $L_{r}-C_{r}$ accounts for the SRR, whereas $C_{g}$ and $C_{p}$ take into account the effect of the slots and the capacitance to ground (losses are not considered in the model since the main aim is to justify the presence of the transmission zero). In order to validate this model, the four reactive parameters must be extracted. To this end, a procedure similar to those reported in [47]-[49] in reference to another type of resonator loaded lines, is considered. Note that four conditions are necessary to unequivocally determine the four model parameters. The first condition is the transmission zero frequency, where the parallel resonant tank opens

$$
f_{z}=\frac{1}{2 \pi \sqrt{L_{r} C_{r}}}
$$

A second condition involving the elements of the series branch is the frequency where such branch shorts, i.e., it exhibits a null reactance. Such frequency, obtained by forcing the reactance of the resonant tank $L_{r}-C_{r}$ plus the capacitance $C_{g}$ to be zero, is 


$$
f_{s}=\frac{1}{\pi \sqrt{2 L_{r}\left(C_{g}+2 C_{r}\right)}}
$$

Note that this frequency can be inferred from the reflection coefficient, $S_{11}$, represented in the Smith chart, since at that frequency the trace of $S_{11}$ intersects the unit conductance circle. From the value of the parallel reactance, directly given by the Smith chart, the shunt capacitance values $\left(C_{p}\right)$ are derived (third condition). Finally, at the reflection zero frequency, $f_{r}$, or frequency with maximum transmission, the iterative impedance, given by

$$
Z_{0}(\omega)=\sqrt{\frac{Z_{s}(\omega) Z_{p}(\omega) / 2}{1+\left(Z_{s}(\omega) / 2 Z_{p}(\omega)\right)}}
$$

must be the reference impedance of the ports $(50 \Omega)$. This is the fourth and last condition, necessary to determine the elements of the circuit model. In (3), $Z_{s}$ and $Z_{p}$ are the impedance of the series and shunt branch of the $\pi$-circuit.

Application of the previous parameter extraction procedure gives the reactive parameters indicated in the caption of Fig. 3 , where the circuit simulation of the frequency response (obtained by means of Keysight ADS) is also depicted. The good agreement between the lossless electromagnetic and circuit simulation validates the proposed circuit model of the SRR-loaded microstrip line (some discrepancies in the vicinity of $f_{z}$ are due to radiation, not accounted for by the circuit model). Note that the considered lossless model is of interest as long as such model predicts the position of the reflection and transmission zero frequencies, relevant to this work. In coherence with the lossless model, parameter extraction has been carried out from the lossless electromagnetic simulation. Parameter extraction from the measured results is also possible, but the inclusion of resistors in the model should be considered (as in [48]). Nevertheless, our purpose has been to provide a lossless model in order to gain insight into the design of the proposed near-field chipless systems.

The frequency response depicted in Fig. 3 corresponds to the SRR-loaded microstrip line without tag loading, i.e., surrounded by air. Let us now consider that the structure is loaded with the tag, and particularly for a tag position corresponding to perfectly aligned SRRs. For this position, referred to as reference position $(R E F)$, the pair of vertically aligned SRRs forms the so-called broadside-coupled SRR (BC-SRR) [46],[47]. This composite particle is characterized by a strong electric coupling between the individual SRRs, provided the air gap separation between them is small enough. The consequence is a significant decrease of the fundamental resonance frequency of the composite particle (BC-SRR), resulting in an overall shift of the transmission coefficient towards lower frequencies. By considering that the air gap separation is $265 \mu \mathrm{m}$ (this value will be justified later), that the SRR of the tag is etched on the Rogers $R O 4003 C$ substrate with thickness $h=0.204 \mathrm{~mm}$, dielectric constant $\varepsilon_{r}=3.55$ and loss tangent $\tan \delta=0.0021$, and that this narrow substrate is attached to FR4 (with thickness $h=1.6 \mathrm{~mm}$, dielectric constant $\varepsilon_{r}=4.7$ and loss tangent $\tan \delta$ $=0.014$ ) for mechanical stability, the resulting frequency response (inferred from electromagnetic simulation) is the one depicted in Fig. 5. Note that if the tag is attached to a

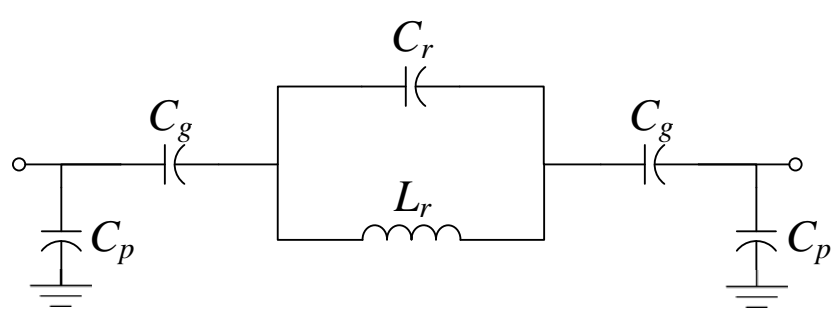

Fig. 4. Lumped element equivalent circuit model of the SRR-loaded microstrip line of Fig. 2.

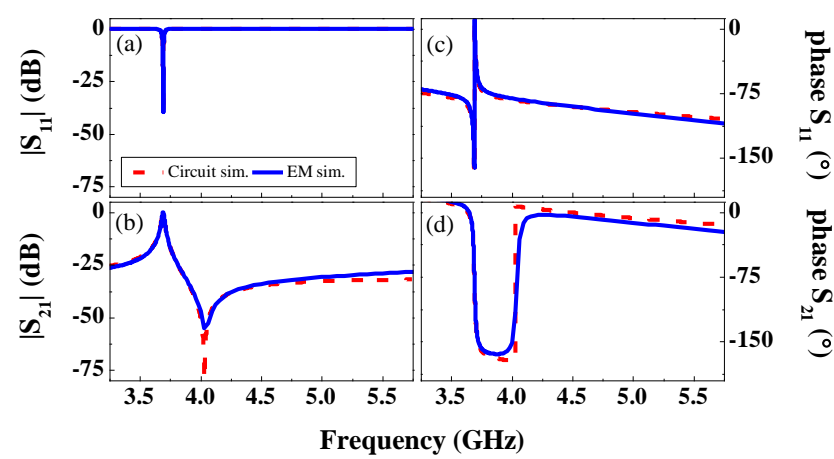

Fig. 5. Frequency response of the structure of Fig. 2 with tag cover as explained in the text, inferred from lossless electromagnetic and circuit simulation. (a) Magnitude of $S_{11}$; (b) magnitude of $S_{21}$; (c) phase of $S_{11}$; (d) phase of $S_{21}$. The extracted parameters of the lumped element equivalent circuit are: $L_{r}=11.9 \mathrm{nH}, C_{r}=0.13 \mathrm{pF}, C_{g}=0.05 \mathrm{pF}$ and $C_{p}=0.87 \mathrm{pF}$.

different material, its effects can be taken into account at simulation and design level. Nevertheless, some tolerance exists in the material used to provide mechanical stability, due to the large excursion of the transmission coefficient experienced by tag motion. The circuit simulation with extracted parameters is also depicted in Fig. 5 (the parameters are indicated in the caption of Fig. 5). Again, good agreement between the lossless circuit and electromagnetic simulation is obtained. Interestingly, $C_{g}$ and $C_{p}$ have not experienced a significant variation as compared to the structure without tag cover, as expected. The main variation corresponds to the capacitance of the resonant element (BC-SRR), which has experienced an increase of roughly four times due to the broadside (face-to-face) capacitance between the metal strips of the particle.

\section{ANALYSIS AND OPTIMIZATION}

The frequency response of the tag loaded structure for different relative positions between the SRR of the tag and the one of the line is depicted in Fig. 6 (tag displacement is in the direction of the line axis). In this case, losses have been considered since the maximum and minimum values of the transmission coefficient are important. The considered air gap separation is the one in reference to the response of Fig. 5 (i.e., $265 \mu \mathrm{m}$ ). As anticipated before, departure from the $R E F$ position modifies (shifts up) the transmission and reflection zero frequencies. Figure 7 depicts the variation of the transmission coefficient as a function of the relative displacement for specific frequencies (indicated in the figure). One of these frequencies is the reflection zero frequency for the case of perfectly aligned SRRs (forming the BC-SRR). Let us designate this frequency as $f_{r, B C-S R R}$. The transmission coefficient for this frequency is a maximum for 


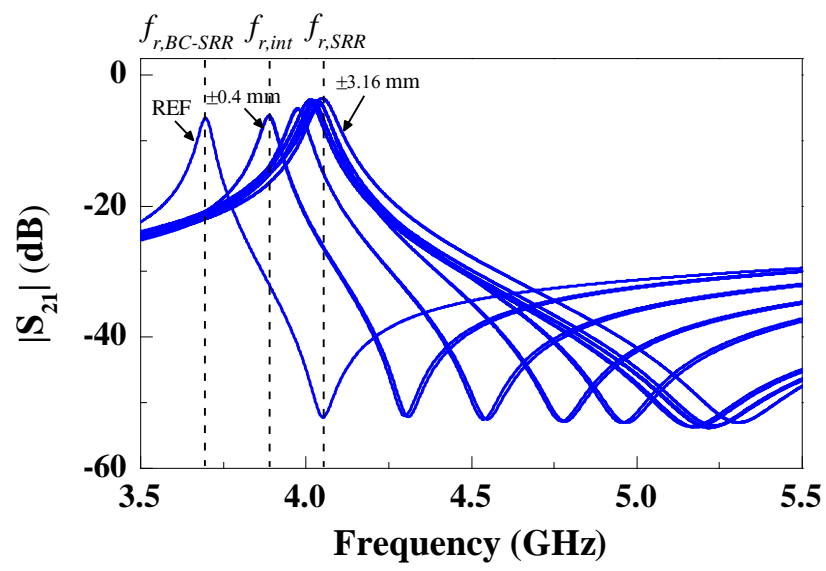

Fig. 6. Transmission coefficient (magnitude) of the SRR-loaded line of Fig. 2 with tag cover, for different relative positions between the SRR of the line and the SRR of the tag. Relevant frequencies, discussed in the text, are indicated. These results have been inferred by electromagnetic simulation.

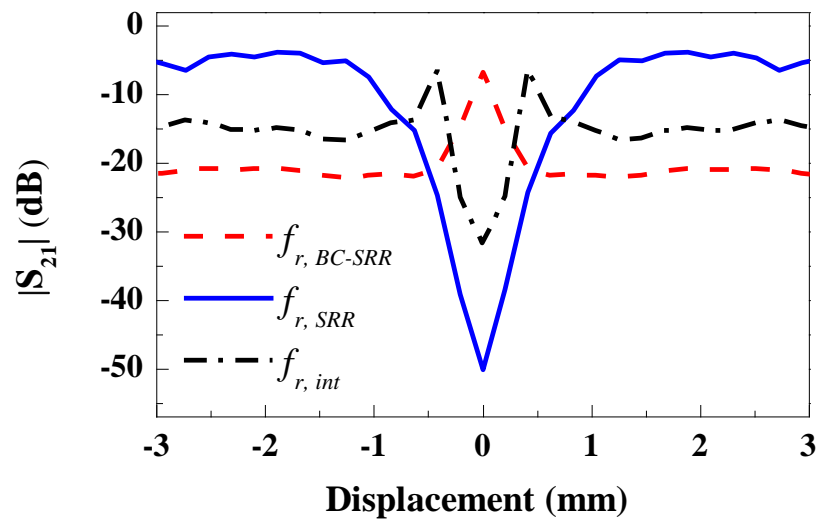

Fig. 7. Variation of the transmission coefficient as a function of the relative displacement for the indicated frequencies. These results have been inferred by electromagnetic simulation.

the $R E F$ position. Departure from this position reduces the transmission coefficient, but it saturates to roughly $-22 \mathrm{~dB}$ for a relatively small displacement. We have also depicted the variation of the transmission coefficient for the reflection zero frequency of the structure with completely misaligned SRRs. Note that this frequency is similar, but not identical, to the reflection zero frequency of the structure without tag loading. The reason is that the presence of the tag substrate slightly modifies the resonance frequency of the SRR of the line. Nevertheless, this frequency is not influenced by the SRR of the tag, and hence it can be called $f_{r, S R R}$ (to point out that this frequency is only given by the SRR of the line). For $f_{r, S R R}$, the excursion (dynamic range) experienced by the transmission coefficient is close to $45 \mathrm{~dB}$, i.e., $-50 \mathrm{~dB}$ for the $R E F$ position, and roughly $-5 \mathrm{~dB}$ for displacements above 1 $\mathrm{mm}$. Finally, we have considered an intermediate frequency, called $f_{r, \text { int }}$, corresponding to the reflection zero frequency for a displacement of $0.4 \mathrm{~mm}$. In this case, the transmission coefficient as a function of the displacement exhibits a deep notch for the $R E F$ position, a maximum for a displacement of $0.4 \mathrm{~mm}$, as expected, and then the transmission coefficient saturates to $-15 \mathrm{~dB}$ as the relative displacement increases.

In view of Fig. 7, it is convenient to set the frequency of the feeding signal, or carrier frequency, to $f_{c}=f_{r, S R R}$. The reason is that for this frequency, the maximum dynamic range is

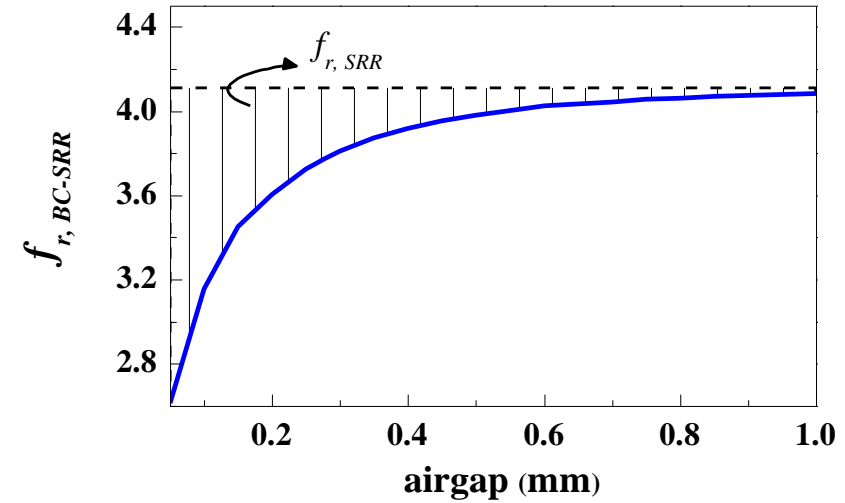

Fig. 8. Variation of $f_{r, B C-S R R}$ with the air gap separation. These results have been inferred by electromagnetic simulation.

obtained. Nevertheless, by choosing the carrier frequency between $f_{r, B C \text {-SRR }}$ and $f_{r, S R R}$, the variation of the transmission coefficient is significant, and the value of the maximum transmission coefficient is close to the ideal value of $0 \mathrm{~dB}$. Note that by setting $f_{c}$ to values above $f_{r, S R R}$ the dynamic range is also considerable, but the value of maximum transmission progressively decreases as $f_{c}$ increases, a situation that must be avoided to prevent deterioration of the modulation index. Thus, according to this analysis, the frequency of the feeding signal (carrier frequency) must satisfy $f_{r, B C-S R R}<f_{c}<f_{r, S R R}$, but, preferably, it must be as close as possible to $f_{r, S R R}$. This window for $f_{c}$ is interesting from a practical viewpoint, since it is difficult to exactly predict the position of the optimum frequency, $f_{r, S R R}$. This analysis reveals that the proposed system is not very sensitive to frequency drifts, this being a relevant aspect.

One important parameter notably influencing the behaviour/performance of the proposed chipless RFID system is the air gap distance. As the gap increases, the SRRloaded line is more insensitive to the presence of the tag, since the coupling between the SRR of the line and the SRR of the tag decreases. By increasing the air gap, $f_{r, B C-S R R}$ increases, whereas $f_{r, S R R}$ remains constant. Figure 8 depicts the variation of $f_{r, B C-S R R}$ with the air gap. It can be seen that the window for $f_{c}$ decreases as the air gap increases. Nevertheless, the window is significant up to reasonable gap distances.

Let us now consider that the carrier frequency is set to the optimum frequency, $f_{c}=f_{r, S R R}$, and let us represent the excursion experienced by the transmission coefficient with the air gap when the SRRs of the line and tag are perfectly aligned. The result, depicted in Fig. 9 (a), reveals that there is an optimum gap spacing, $265 \mu \mathrm{m}$, providing the maximum variation of the transmission coefficient. The particularity of this gap separation is that, for perfectly aligned SRRs, the transmission zero frequency exactly coincides with the reflection zero frequency of the structure without tag on top of it, i.e., $f_{r, S R R}$, which is in turn the carrier frequency. It should be highlighted, however, that this optimum gap space has been calculated for a particular carrier frequency, i.e., $f_{c}$ $=f_{r, S R R}$. If the carrier frequency is slightly below $f_{r, S R R}$, then it is necessary to decrease the gap in order to allocate the transmission zero, for the perfectly aligned SRRs, at the position of the carrier frequency. Thus, the optimum gap separation depends on the carrier frequency, but such optimum gap separation increases with $f_{c}$. Since $f_{c}$ should not 
(a)

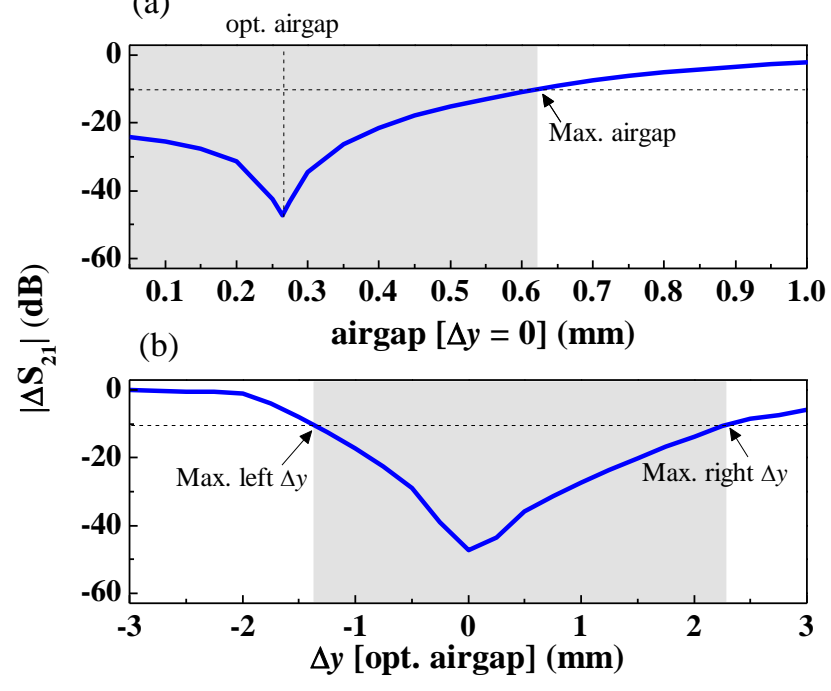

Fig. 9. Tolerance analysis for vertical and lateral displacement of the tag on the maximum variation of the transmission coefficient. (a) Effects of air gap variation; (b) effects of lateral shift. The mechanical system used to provide relative motion between tag and reader must guarantee lateral and vertical misalignments within the indicated tolerance limits. These results have been inferred by electromagnetic simulation.

be chosen to be higher than $f_{r, S R R}$, it follows that $f_{r, S R R}$ is the optimum carrier frequency, provided the optimum gap distance is the largest one within the interval. Nevertheless, small variations of $f_{c}$ in the vicinity of $f_{r, S R R}$ (with the gap set to the optimum value at $f_{r, S R R}$ ) do also give significant excursions in the transmission coefficient.

In order to analyze the tolerance against lateral shifts of the tag with regard to the line axis, Fig. 9(b) depicts the maximum variation of the transmission coefficient for the optimum frequency $f_{r, S R R}$ and the optimum gap separation at this frequency $(265 \mu \mathrm{m})$. As can be seen, laterally shifting the tag degrades the excursion of the transmission coefficient. However, by considering a tolerance limit of -10 $\mathrm{dB}$ for a reliable reading operation, it follows that lateral shifts between $-1.3 \mathrm{~mm}$ and $+2.3 \mathrm{~mm}$ are within the allowable limits for misalignment in the transverse direction. These values are very reasonable on account of the considered SRR dimensions. It is worth mentioning that the tolerance interval is not symmetric. The reason is the lack of symmetry of the structure with regard to the line axis. However, due to the symmetry with regard to the midplane between the input and the output port for perfectly aligned SRRs, it follows that tag displacement in the positive or negative direction, from the $R E F$ position, along the line axis is undistinguishable. For this reason, the curves of Fig. 7 exhibit perfect symmetry.

\section{FABRICATED TAGS, EXPERIMENTAL SET-UP AND RESULTS}

The in-house system for the measurement of the tag response is based on a step motor that provides angular motion to a rotor. For this reason, the fabricated chipless RFID tags have been implemented as circular chains of SRRs. In particular, we have considered four different 40-bit tags based on the SRRs considered in the previous sections (see the dimensions in Fig. 2) and implemented on a narrow substrate attached to FR4 for mechanical stability (see

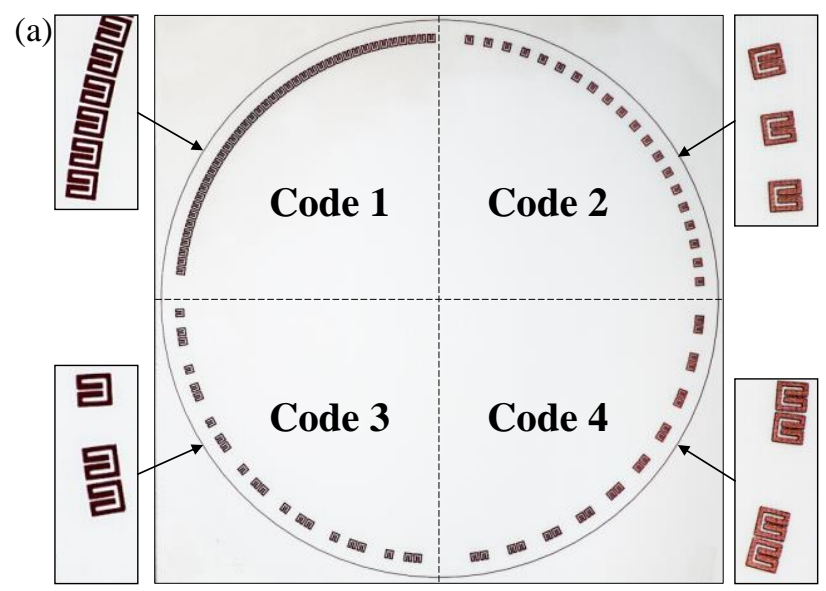

(b)

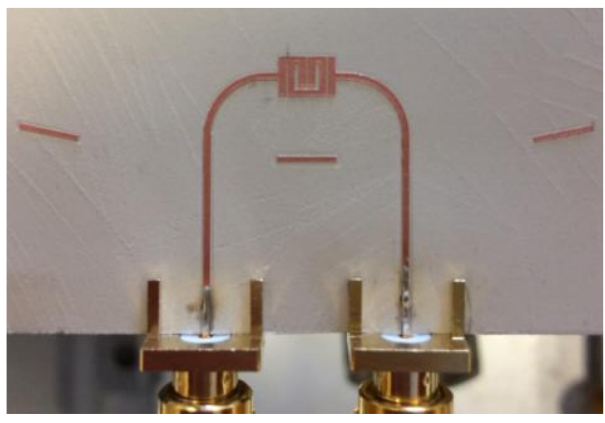

Fig. 10. Photograph of the fabricated (a) 40-bit chipless RFID tags and (b) active part of the reader.

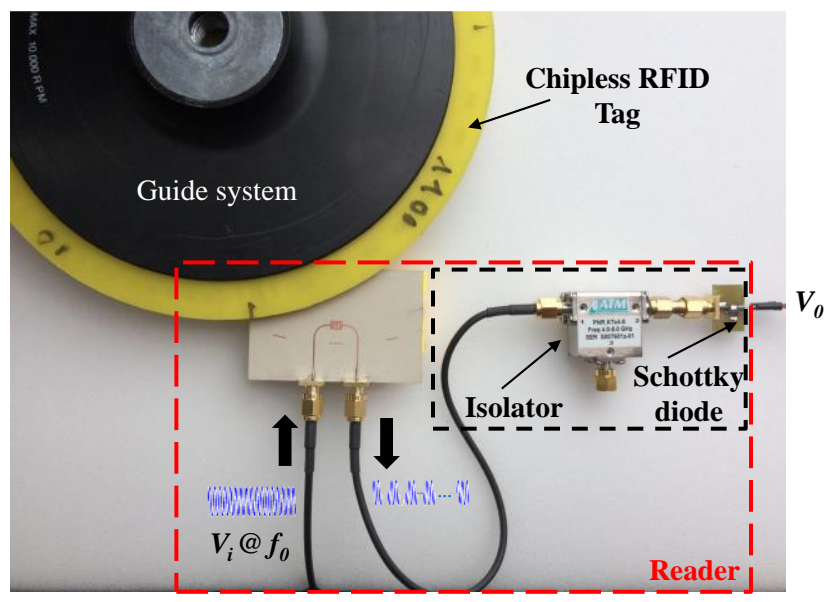

Fig. 11. Experimental set-up used for tag reading.

Section III in reference to Fig. 5). Such tags are allocated in the four quadrants of a circle [see Fig. 10(a)]. However, the whole structure can be viewed as a single 160-bit tag as well. The photograph of the SRR-loaded line (active part of the reader) is depicted in Fig. 10(b). Note that the $50 \Omega$ access lines have been bent to avoid mechanical friction between the port connectors and the tag during tag motion.

The photograph of the experimental set-up is shown in Fig. 11. The Agilent E4438C function generator has been used to feed the SRR-loaded line through a harmonic signal tuned to $f_{c}=f_{r, S R R}=4 \mathrm{GHz}$. This frequency provides reasonable SRR size and it is compatible with our measurement equipment, and system components. The envelope detector has been implemented by means of a Shottky diode (Avago HSMS-2860) and a low pass filter. In practice, the low pass filter functionality has been achieved by means of an active probe (N2795A), with resistance 


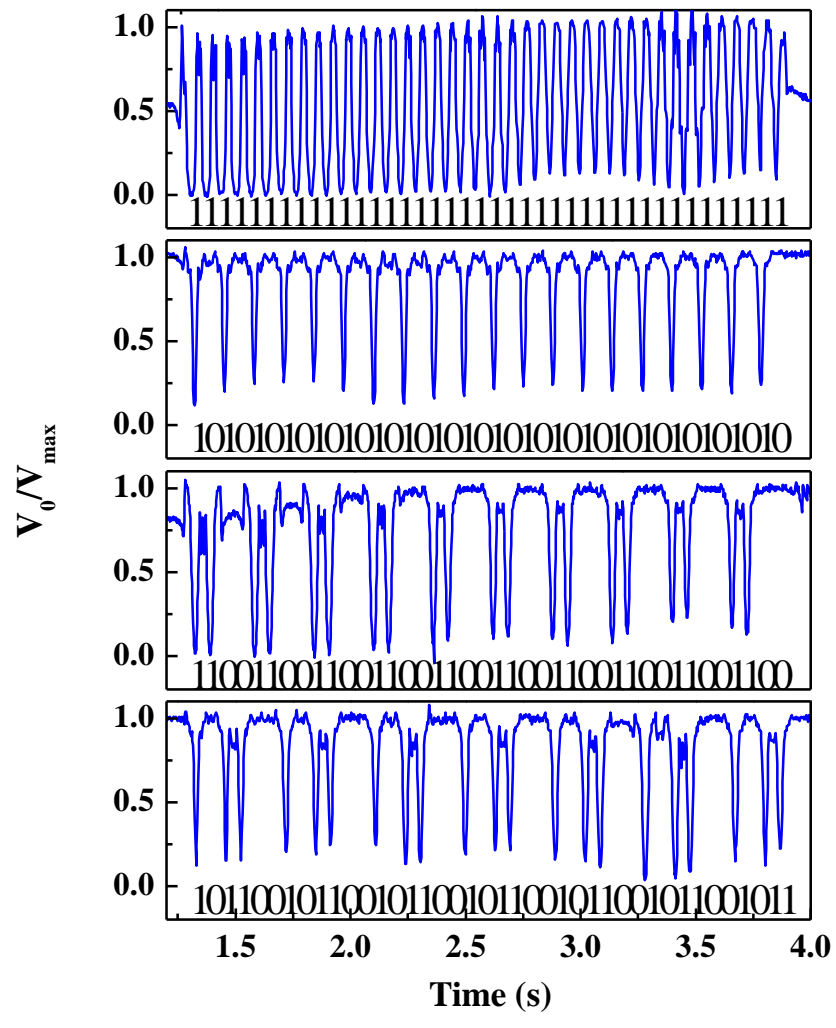

Fig. 12. Measured normalized envelope for the 40-bit fabricated tags The depth of the dips, corresponding to the logic state ' 1 ', is significantly larger as compared to the system in [41].

$R=1 \mathrm{M} \Omega$ and capacitance $C=1 \mathrm{pF}$, connected to an oscilloscope. Such oscilloscope (the Agilent MSO-X-3104A) is used to visualize the envelope function, providing the tag ID code. In order to avoid unwanted reflections from the Schottky diode (a highly nonlinear device), a circulator (model ATM ATc4-8), configured as an isolator, has been cascaded between the output port of the SRR-loaded line and the Shottky diode, similar to the system in [41]. Finally, the step motor STM $23 Q-3 A N$ is used to provide angular motion to the tags. The response of the four fabricated chipless tags is depicted in Fig. 12, where the ID codes are indicated. The logic states ' 1 ' and ' 0 ' are given by the presence and absence, respectively, of SRRs at the predefined positions, as mentioned before. The angular velocity of the step motor has been set to $5.33 \mathrm{rpm}$. It can be seen that the notches in the time response perfectly correlate with the logic state ' 1 '. The significant dip depth indicates that the reader is very sensitive for the detection of the logic state ' 1 ' and hence it is a robust system for tag identification. Thus, the results presented in Fig. 12 validate the proposed approach for the determination of the tag ID codes. The area of each 40-bit tag is as small as $4.75 \mathrm{~cm}^{2}$ (corresponding to a density of $8.4 \mathrm{bit} / \mathrm{cm}^{2}$ ). Note that by increasing the number of bits of the tags, the time required to read a tag also increases. However, in practice, the tag reading speed may be large since the carrier frequency ( $4 \mathrm{GHz}$ ) is very large as compared to the rhythm of SRR crossing above the reader, imposed by any reasonable, but large, shifting speed of the tag. Tag displacement speed does not have any influence on system performance, since, as mentioned, any reasonable speed is necessarily small as compared to the carrier frequency. Nevertheless, in a real scenario, tag speed is actually limited by the sampling time

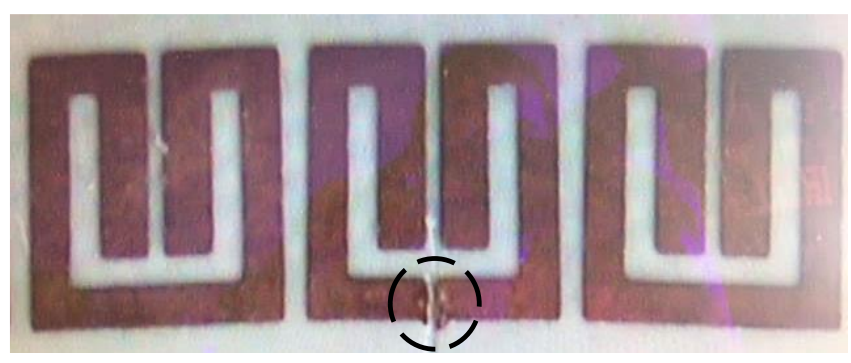

Fig. 13. Photograph of the detuned SRR

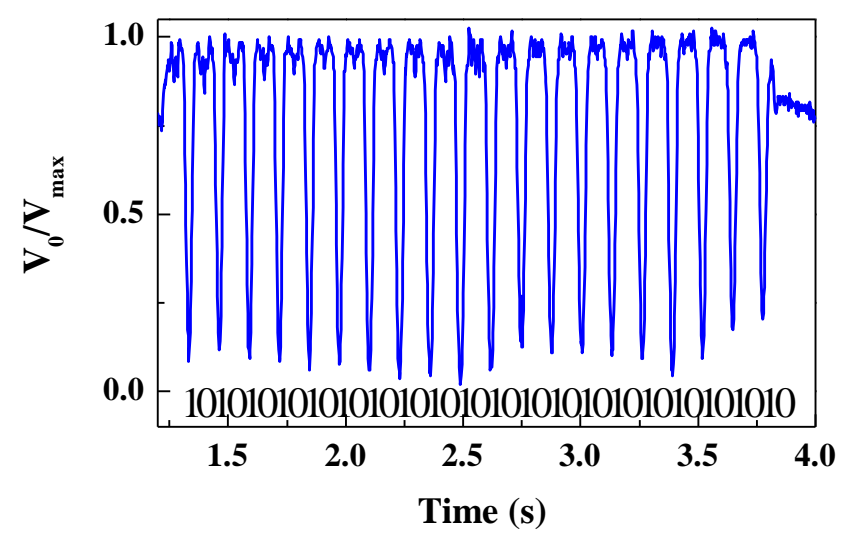

Fig. 14. Measured normalized envelope for the 40-bit programmed tag.

of the data acquisition system, which must be significantly smaller than the temporal width of the dips.

\section{DISCUSSION}

An important aspect of chipless-RFID systems, with direct impact on cost, concerns the possibility of implementing programmable tags. As we have previously discussed, the presence or absence of resonant elements at the predefined tag positions determines the logic state of each bit. Note, however, that this approach is not optimum from the point of view of overall costs. In a real scenario, where tags are implemented by printing processes using conductive inks, the cost of the necessary ink for tag fabrication is small (calculated in the range of less than one eurocent) as compared to the price of typical RFID chips. However, each ID code requires a specific layout, and this may represent a significant cost burden, especially if high-quality massive printing processes are required for tag fabrication (e.g. rotogravure).

An alternative approach, of special interest if thousands of high-data capacity tags are necessary, is to fabricate allidentical tags with all the resonators printed at the predefined positions (representing an optimum cost solution). Tag encoding (programming) can then be done in a later (lowcost) stage by making inoperative those resonant elements with required ' 0 ' state. In this context, inoperative resonators means detuned resonators, i.e., with fundamental resonance significantly shifted. Resonator detuning can be achieved, e.g., by short-circuiting the resonant elements (an approach proposed in [17] for frequency-domain multi-resonator spectral signature barcodes) or by physically cutting the resonant elements. In a real scenario, short-circuiting can be achieved by inkjet printing (requiring a minimum quantity of ink and sintering at room temperature), whereas resonator cutting can be achieved, for instance, by laser ablation. 


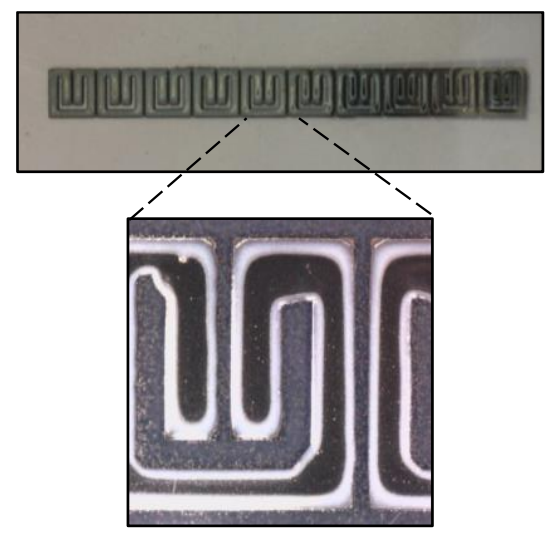

Fig. 15. Photograph of the 10-bit printed chipless tag implemented on PEN substrate.

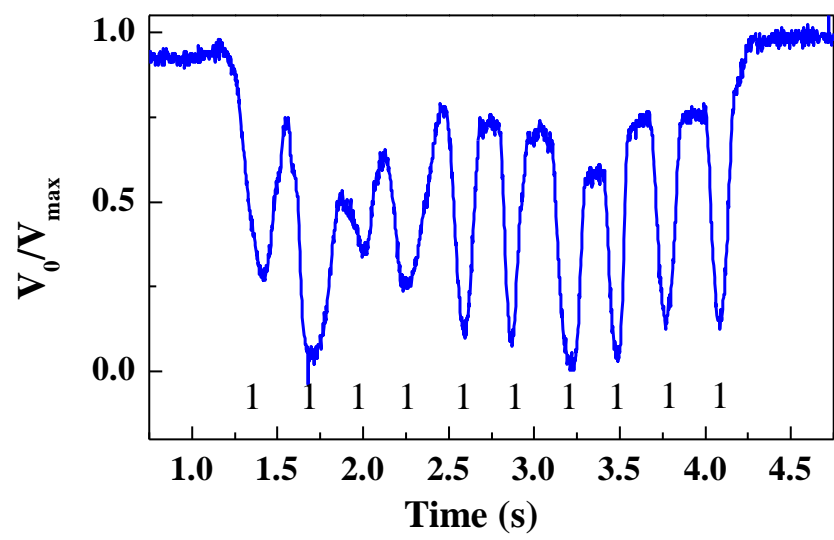

Fig. 16. Measured normalized envelope for the 10-bit programmed tag.

In this paper, a proof-of-concept to demonstrate the programmable capability of the proposed chipless-RFID tags has been carried out. To this end, the 40-bit encoder with all resonators present at the predefined positions (code 1 of Fig. 10) has been modified (programmed). Specifically, we have cut alternate resonators, making them inoperative and hence programming the ID code corresponding to code 2 of Fig. 10. A drilling machine has been used to cut the required resonant elements along their symmetry plane (the photograph of one of such detuned resonators is depicted in Fig. 13). The measured normalized envelope function corresponding to this programmed tag is depicted in Fig. 14, where it can be appreciated that the 40-bit ID code is given by alternating states ' 1 ' and ' 0 ', as expected.

The functionality of the proposed chipless-RFID system has been demonstrated by tags implemented in a commercial (narrow) low-loss microwave substrate. However, the interest in a real application devoted to security and/or authentication is the implementation of these chipless-RFID tags on low-cost plastic or paper substrates through standard printing processes (e.g., rotogravure, screen-printing, etc.), and, eventually, programming in a later stage, as discussed before. Note that in applications such as secure paper (e.g. for anti-counterfeiting of corporate documents, certificates, ballots, etc.), the ideal solution is to directly print the tags on the tagged item (paper). Alternatively, a plastic substrate, with intermediate electromagnetic properties between commercial low-loss microwave substrates and paper, located in a specific region of the tagged item, can be considered for tag printing.

As a first proof-of-concept towards this direction, we have fabricated, by inkjet printing, a 10-bit tag with all bits set to
' 1 ' (all resonators present and functional) on the Polyethilene naphthalate (PEN) film (Dupont Teijin Q65FA) (Fig. 15). The used inkjet printer is the Ceradrop CeraPrinter $X$-Serie, and two layers of DuPont ${ }^{\mathrm{TM}}$ PE410 conductive ink (with a measured conductivity of $7.28 \times 10^{6} \mathrm{~S} / \mathrm{m}$ ) have been printed in order to achieve a measured thickness of 3.3-3.5 $\mu \mathrm{m}$. The considered substrate (PEN) has a measured thickness, dielectric constant and loss tangent of $h=0.125 \mathrm{~mm}$, $\varepsilon_{r}=3.36$ and $\tan \delta=0.0042$, respectively. For tag reading, the tag has been attached to the FR4 substrate to provide mechanical stability.

Since the SRR chain is linear (see Fig. 15), rather than using the step motor, we have linearly displaced the tag over the SRR of the line (reader) through our available positioning system, which allows for manual linear motion in two dimensions $(x, y)$. Apart from that, the setup for tag reading (i.e., for obtaining the envelope function) is identical to that described in Section V. The measured envelope function corresponding to the tag of Fig. 15 is depicted in Fig. 16. Ten dips, corresponding to the ten SRRs, can be perfectly appreciated (the lower depth of the $2 \mathrm{~s}$ dip is due to fabrication related tolerances; nevertheless the ID code can be perfectly identified). Hence, the proposed chipless RFID system is validated by considering plastic substrates and resonant elements printed on it.

We would like also to highlight that the proposed chiplessRFID system is conceived by taking into account that the relative speed between the tag and the reader should be constant. However, different (constant) velocities can be considered, preferably high in order to reduce the time needed for tag reading. In order to know such velocity, necessary for tag reading, one possibility is to add a pair of symbols (SRRs) at the beginning of the chain. The time distance between these pair of symbols, read as logic ' 1 ', determine the time positions where tag must be read.

As previously mentioned, the main advantage of chipless RFID over RFID systems with tags equipped with chips is the lower cost of the chipless tags. In many applications involving thousands or millions of tags, or in order to tag low cost items, chipless RFID is fully justified even at the expense of potentially higher cost in the reader side (in our case due to the mechanical elements needed to provide tag motion). Nevertheless, concerning the electronics of the reader of the proposed system, it is relatively low cost. Note that in a real scenario, the function generator should be replaced by a harmonic oscillator, and the information contained in the tag can be inferred by means of a low cost post-processing unit (out of the scope of this paper). Potential applications of the proposed near-field chipless-RFID system in the field of security and authentication include authentication and identification of corporate documents, ballots, exams, certificates, etc.

\section{CONCLUSIONS}

In conclusion, we have presented a chipless RFID system based on near field coupling and sequential bit reading that constitutes an improvement as compared to the first version reported in [41]. The tags are simply a set of identical resonant elements (SRRs) etched or printed on a dielectric substrate forming a chain, and the presence or absence of resonant elements at predefined positions in the chain determines the logic state. Reading is achieved by proximity between the tag and the reader, a SRR-loaded microstrip line fed by a harmonic signal (carrier). Specifically, it has been 
demonstrated that by displacing the tag (SRR chain) above the SRR of the line, the transmission coefficient is efficiently modulated, and this modulation is dictated by the presence/absence of SRRs in chain. The result is that the amplitude of the carrier signal is modulated at the output port of the microstrip line, and the ID code is contained in the envelope function. The main advantages of the proposed approach as compared to chipless RFID system proposed in [41] are: (i) vias are not required, since a microstrip line, rather an a CPW, is used as active part of the reader; (ii) backside isolation in the reader is guaranteed by the presence of the ground plane in the back substrate side, (iii) the modulation index is high due to the high excursion of the transmission coefficient with the relative position between the tag and the SRR-loaded line. Moreover, it has been found that the system is tolerant to air gap variations (distance between the tag and the SRR of the reader) up to $0.6 \mathrm{~mm}$, and to lateral tag shifts up to $1.3 \mathrm{~mm}$, which are very reasonable values on account of the dimensions of the tag SRRs. The number of bits of the proposed system is only limited by the space occupied by the tag chain, since tag reading simply requires a harmonic (single-tone continuous-wave) signal. The achieved density of bits per area is as high as $8.4 \mathrm{bits} / \mathrm{cm}^{2}$. Therefore, high data capacity (at the expense of reading by proximity), useful in applications such as security and authentication, is achievable. Particularly, applications in secure paper, where the tag ID can be directly printed on a paper substrate, and reading can be merely achieved by a mechanical system able to provide tag motion above the reader (plus the necessary electronics), are envisaged.

\section{REFERENCES}

[1] S. Preradovic and N. C. Karmakar, "Chipless RFID: bar code of the future," IEEE Microwave Magazine, vol. 11, pp. 87-97, 2010

[2] S. Preradovic and N. C. Karmakar, Multiresonator-Based Chipless RFID: Barcode of the Future, Springer, 2011.

[3] N.C. Karmakar, R. Koswatta, P. Kalansuriya, R. E-Azim, Chipless RFID Reader Architecture, Artech House, 2013.

[4] E. Perret, Radio Frequency Identification and Sensors: From RFID to Chipless RFID, John Wiley, New York, 2014.

[5] R. Rezaiesarlak, M. Manteghi, Chipless RFID: Design Procedure and Detection Techniques, Springer, 2015.

[6] N. C. Karmakar, M. Zomorrodi, C. Divarathne, Advanced Chipless RFID, John Wiley, Hoboken, NJ, 2016.

[7] C. S. Hartmann, "A global SAW ID tag with large data capacity," in Proc. of IEEE Ultrasonics Symposium, October 2002, vol. 1, pp. 6569.

[8] A. Chamarti and K. Varahramyan, "Transmission delay line based ID generation circuit for RFID applications," IEEE Microw. Wireless Compon. Lett., vol. 16, pp. 588-590, 2006.

[9] M. Schüßler, C. Damm, and R. Jakoby, "Periodically LC loaded lines for RFID backscatter applications," in Proc. of Metamaterials 2007, Rome, Italy, October 2007, pp. 103-106.

[10] N. Saldanha, D.C. Malocha, "Design Parameters for SAW multi-tone frequency coded reflectors" 2007 IEEE Ultrasonics Symp., pp. 2087. 2090, 2007.

[11] M. Schüßler, C. Damm, M. Maasch, and R. Jakoby, "Performance evaluation of left-handed delay lines for RFID backscatter applications," in Proc. of the IEEE MTT-S International Microwave Symposium 2008, pp. 177-180.

[12] S. Harma, V.P. Plessky, C.S. Hartmann, W. Steichen, "Z-path SAW RFID tag" IEEE Trans. Ultrasonics, Ferroelectric Freq. Control, vol. 55, pp. 208-213, 2008.

[13] H. Tao, W. Weibiao, W. Haodong, S. Yongan, "Reflection and scattering characteristics of reflectors in SAW tags", IEEE Trans. Ultrasonics, Ferroelectric Freq. Control, vol. 55, pp. 1387-1390, 2008.

[14] S. Harma, V.P. Plessky, L. Xianyi, P. Hartogh, "Feasibility of ultrawideband SAW RFID tags meeting FCC rules" IEEE Trans. Ultrasonics, Ferroelectric Freq. Control, vol. 56, pp. 812-820, 2012.

[15] F.J. Herraiz-Martínez, F. Paredes, G. Zamora, F. Martín, and J. Bonache, "Printed magnetoinductive-wave (MIW) delay lines for chipless RFID applications", IEEE Trans. Ant. Propag., vol. 60, pp. 5075-5082, Nov. 2012.

[16] S. Tedjini, E. Perret, A. Vena, D. Kaddout, "Mastering the electromagnetic signature of chipless RFID tags", in Chipless and Conventional Radiofrequency Identification, ed. IGI Global, 2012.

[17] S. Preradovic, I. Balbin, N. C. Karmakar, and G. F. Swiegers, "Multiresonator-based chipless RFID system for low-cost item tracking," IEEE Trans. Microw. Theory Techn., vol. 57, pp. 14111419, 2009.

[18] S. Preradovic and N. C. Karmakar, "Design of chipless RFID tag for operation on flexible laminates," IEEE Anten. Wireless Propag. Lett., vol. 9, pp. 207-210, 2010.

[19] O. Rance, R. Siragusa, P. Lemaître-Auger, E. Perret, "Toward RCS magnitude level coding for chipless RFID," IEEE Trans. Microw. Theory Techn., vol. 64, pp. 2315-2325, Jul. 2016.

[20] J. McVay, A. Hoorfar, and N. Engheta, "Space-filling curve RFID tags," in Proc. of 2006 IEEE Radio Wireless Symp., pp. 199-202.

[21] I. Jalaly and D. Robertson, "Capacitively-tuned split microstrip resonators for RFID barcodes," in Proc. of European Microwave Conference, October 2005, vol. 2, pp. 4-7.

[22] H.-S. Jang, W.-G. Lim, K.-S. Oh, S.-M. Moon, and J.-W. Yu, "Design of low-cost chipless system using printable chipless tag with electromagnetic code", IEEE Microw. Wireless Compon. Lett., vol. 20, pp. 640-642, 2010

[23] A. Vena, E. Perret, and S. Tedjini, "A fully printable chipless RFID tag with detuning correction technique", IEEE Microw. Wireless Compon. Lett., vol. 22(4), pp. 209-211, 2012

[24] A. Vena, E. Perret, and S. Tedjini, "Design of compact and autocompensated single-layer chipless RFID tag", IEEE Trans. Microw. Theory Techn., vol. 60(9), pp. 2913-2924, Sept. 2012.

[25] A. Vena, E. Perret, and S. Tedjini, "High-capacity chipless RFID tag insensitive to the polarization", IEEE Trans. Ant. Propag., vol. 60(10), pp. 4509-4515, Oct. 2012.

[26] M. M. Khan, F. A. Tahir, M. F. Farooqui, A. Shamim, H. M. Cheema, "3.56-bits $/ \mathrm{cm}^{2}$ compact inkjet printed and application specific chipless RFID tag," IEEE Ant. Wireless Propag. Lett., vol. 15, pp. 1109-1112, 2016.

[27] M. A. Islam and N. C. Karmakar, "A novel compact printable dualpolarized chipless RFID system," IEEE Trans. Microw. Theory Techn., vol. 60, pp. 2142-2151, Jul. 2012.

[28] R. Rezaiesarlak and M. Manteghi, "Complex-natural-resonance-based design of chipless RFID tag for high-density data," IEEE Trans. Ant. Propag., vol. 62, pp. 898-904, Feb. 2014.

[29] M. Svanda, J. Machac, M. Polivka, J. Havlicek., "A comparison of two ways to reducing the mutual coupling of chipless RFID tag scatterers," in Proc. of 21st International Conference on Microwave, Radar and Wireless Communications (MIKON), May 2016, pp. 1-4.

[30] A. Vena, E. Perret, S. Tedjini, "Chipless RFID tag using hybrid coding technique," IEEE Trans. Microw. Theory Techn., vol. 59, pp. 33563364, Dec. 2011

[31] A. Vena, E. Perret, S. Tedjini, "A compact chipless RFID tag using polarization diversity for encoding and sensing", 2012 IEEE Int. Conf. RFID, pp. 191-197, 2012

[32] I. Balbin, N.C. Karmakar, "Phase-encoded chipless RFID transponder for large scale low cost applications", IEEE Microw. Wireless. Comp. Lett., vol. 19, pp. 509-511, 2009.

[33] S. Genovesi, F. Costa, A. Monorchio, G. Manara, "Chipless RFID tag exploiting multifrequency delta-phase quantization encoding", IEEE Ant. Wireless, Propag. Lett., vol. 15, pp. 738-741, 2015.

[34] C. Herrojo, J. Naqui, F. Paredes and F. Martín, "Spectral Signature Barcodes based on S-shaped Split Ring Resonators (S-SRR)", EPJ Applied Metamaterials, vol. 3, pp. 1-6, June 2016.

[35] C. Herrojo, J. Naqui, F. Paredes, F. Martín, "Spectral signature barcodes implemented by multi-state multi-resonator circuits for chipless RFID tags", IEEE MTT-S International Microwave Symposium (IMS'16), San Francisco, May 2016.

[36] C. Herrojo, F. Paredes, J. Mata-Contreras, S. Zuffanelli and F. Martín, "Multi-state multi-resonator spectral signature barcodes implemented by means of S-shaped Split Ring Resonators (S-SRR)", IEEE Trans. Microw. Theory Techn., vol. 65, no. 7, pp. 2341-2352 July 2017.

[37] H. Chen, L. Ran, J. Huangfu, X. Zhang, K. Chen, T. M. Grzegorczyk, and J. A. Kong, "Left-handed materials composed of only S-shaped resonators," Phys. Rev. E, vol. 70, no. 5, p. 057605, Nov. 2004.

[38] H. Chen, L. Ran, J. Huangfu, X. Zhang, K. Chen, T. M. Grzegorczyk, and J. A. Kong, "Negative refraction of a combined double S-shaped metamaterial," Appl. Phys. Lett., vol. 86, no. 15, p. 151909, 2005. 
[39] H. Chen, L.-X. Ran, H.-F. Jiang Tao, X.-M. Zhang, K.-S. Cheng, T. M Grzegorczyk, and J. A. Kong, "Magnetic properties of S-shaped split ring resonators," Prog. Electromagn. Res., vol. 51, pp. 231-247, 2005.

[40] J. Naqui, J. Coromina, A. Karami-Horestani, C. Fumeaux, and F. Martín, "Angular displacement and velocity sensors based on coplanar waveguides (CPWs) loaded with S-shaped split ring resonator (SSRR)", Sensors, vol. 15, pp. 9628-9650, 2015.

[41] C. Herrojo, J. Mata-Contreras, F. Paredes, Ferran Martín, "Near-Field chipless RFID encoders with sequential bit reading and high data capacity", IEEE MTT-S Int. Microw. Symp. (IMS'17), Honolulu, Hawaii, June 2017.

[42] J. Naqui, F. Martín, "Application of broadside-coupled split ring resonator (BC-SRR) loaded transmission lines to the design of rotary encoders for space applications", IEEE MTT-S Int. Microw. Symp. (IMS'16), San Francisco, May 2016.

[43] J. Mata-Contreras, C. Herrojo, and F. Martín, "Application of split ring resonator (SRR) loaded transmission lines to the design of angular displacement and velocity sensors for space applications", IEEE Trans. Microw. Theory Techn., published online (doi 10.1109/TMTT.2017.2693981).

[44] J.B. Pendry, A.J. Holden, D.J. Robbins and W.J. Stewart, "Magnetism from conductors and enhanced nonlinear phenomena", IEEE Transactions Microwave Theory Tech., vol. 47, pp. 2075-2084 Nov. 1999.

[45] F. Martín, F. Falcone, J. Bonache, R. Marqués and M. Sorolla, "Split ring resonator based left handed coplanar waveguide", Appl. Phys. Lett., vol. 83, pp. 4652-4654, Dec. 2003.

[46] R. Marques, F. Medina and R. Rafii-El-Idrissi, "Role of bi-anisotropy in negative permeability and left handed metamaterials", Phys. Rev. B vol. 65, paper 144441, 2002.

[47] F. Martin, Artificial Transmission Lines for $R F$ and Microwave Applications, John Wiley, Hoboken, NJ, USA, 2015.

[48] J. Bonache, M. Gil, I. Gil, J. Garcia-García and F. Martín, "On the electrical characteristics of complementary metamaterial resonators", IEEE Microw. Wireless Compon. Lett., vol. 16, pp. 543.545, Oct. 2006.

[49] F. Aznar, M. Gil, J. Bonache, J.D. Baena, L. Jelinek and R. Marqués and F. Martín, "Characterization of miniaturized metamaterial resonators coupled to planar transmission lines" J. Appl. Phys., vol. 104, paper 114501-1-8, Dec. 2008

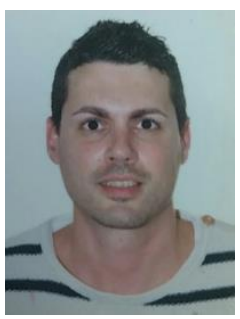

Cristian Herrojo was born in Badalon (Barcelona), Spain, in 1983. He received the Telecommunications Technical Engineering degree (specialty in Electronic Systems) and the Telecommunications Engineering degree in 2010 and 2012, respectively. Currently, he is working toward his $\mathrm{PhD}$ which is mainly focused on the design of RF/microwave resonant structures applied to RFID tags (Radio Frequency Identification) without chip and he has a research grant from FPI Program of the Education and Science Spanish Ministry.

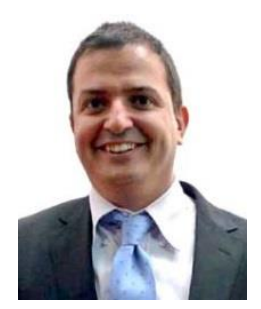

Javier Mata-Contreras was born in 1976 in Málaga (Spain). He received the Ingeniería de Telecomunicación Degree from the Universidad de Málaga (UMA) in 2000 and the PhD degree from the same university in 2010, with the Thesis "Distributed Amplifiers and Mixers with Transmission Lines based on Metamaterials". In 2000, he joined the UMA Department of Ingeniería de Comunicaciones UMA as Assistant Professor. He is currently working at CIMITEC and the Universitat Autònoma de Barcelona as Visitant Professor. His research interests include active and passive microwave devices and active distributed circuits based on metamaterials, among others.

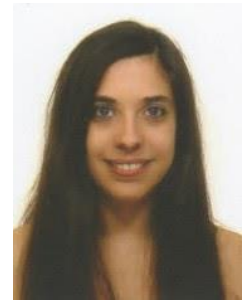

Alba Nuñez was born in Madrid, Spain, in 1991. She received the Physics Science degree from the Universidad Complutense de Madrid in 2014 and the Master degree in Advanced Materials and Nanophysics in 2015 from the same University. In 2016, she joined the Printed Electronic Group at the Institute of Microelectronics of Barcelona IMB CNM (CSIC). Currently, she is a research technician specialized in the fabrication of inkjet printed devices.

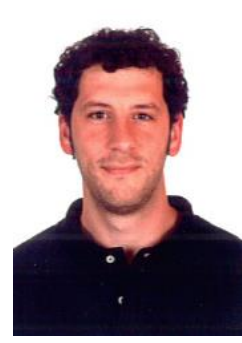

Ferran Paredes was born in Badalona (Barcelona), Spain in 1983. He received the Telecommunications Engineering Diploma (specializing in Electronics) and the Telecommunications Engineering degree from the Universitat Autònoma de Barcelona in 2004 and 2006, respectively and the $\mathrm{PhD}$ degree in Electronics Engineering from the same university in 2012. He was Assistant Professor from 2006 to 2008 at the Universitat Autònoma de Barcelona, where he is currently working as a Research Assistant. His research interests include metamaterial concepts, passive microwaves devices, antennas and RFID.

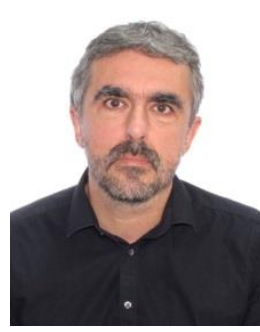

Eloi Ramon graduated in Telecom Engineering from the Polytechnic University of Catalonia (UPC), holds a Master in Micro- and Nanoelectronics Engineering and a PhD on inkjet printed devices and circuits from the Autonomous University of Barcelona (UAB). Since 1999, he is an Associate Professor at the Electronic Dept. (UAB) where he is teaching Telecom and CS BsC and MA. In 2014 he joined IMB-CNM (CSIC) as Printed Microelectronics researcher \& group leader of Printed Electronics R\&D line. His work is focused in the area of printed \& organic devices for electronic systems, radiofrequency and biomedical applications, with large focus on the application of inkjet printing technologies for functional devices manufacturing. He has participated in more than 55 industrial and research projects, being co-author of more than 40 papers and 50 conference presentations and serving as a reviewer and scientific expert for different journals and public funding agencies.

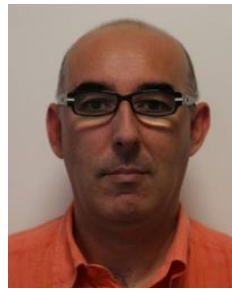

Ferran Martín (M'04-SM'08-F'12) was born in Barakaldo (Vizcaya), Spain in 1965. He received the B.S. Degree in Physics from the Universita Autònoma de Barcelona (UAB) in 1988 and the $\mathrm{PhD}$ degree in 1992. From 1994 up to 2006 he was Associate Professor in Electronics at the Departament d'Enginyeria Electrònica (Universitat Autònoma de Barcelona), and since 2007 he is Ful Professor of Electronics. In recent years, he has been involved in different research activities including modelling and simulation of electron devices for high frequency applications, millimeter wave and $\mathrm{THz}$ generation systems, and the application of electromagnetic bandgaps to microwave and millimeter wave circuits. He is now very active in the field of metamaterials and their application to the miniaturization and optimization of microwave circuits and antennas. He is the head of the Microwave Engineering, Metamaterials and Antennas Group (GEMMA Group) at UAB, and director of CIMITEC, a research Center on Metamaterials supported by TECNIO (Generalitat de Catalunya). He has organized several international events related to metamaterials, including Workshops at the IEEE International Microwave Symposium (years 2005 and 2007) and European Microwave Conference (2009), and the Fifth International Congress on Advanced Electromagnetic Materials in Microwaves and Optics (Metamaterials 2011), where he has acted as chair of the Local Organizing Committee. He has acted as Guest Editor for three Special Issues on Metamaterials in three International Journals. He has authored and co-authored over 500 technical conference, letter, journal papers and book chapters, he is co-author of the book on Metamaterials entitled Metamaterials with Negative Parameters: Theory Design and Microwave Applications (John Wiley \& Sons Inc. 2008), author of the book Artificial Transmission Lines for $R F$ and Microwave Applications (John Wiley \& Sons Inc. 2015), and he has generated 17 PhDs. Ferran Martín has filed several patents on metamaterials and has headed several Development Contracts.

Prof. Martín is a member of the IEEE Microwave Theory and Techniques Society (IEEE MTT-S). He is reviewer of the IEEE Transactions on Microwave Theory and Techniques and IEEE Microwave and Wireless Components Letters, among many other journals, and he serves as member of the Editorial Board of IET Microwaves, Antennas and Propagation and International Journal of RF and Microwave Computer-Aided Engineering. $\mathrm{He}$ is also a member of the Technical Committees of the European Microwave Conference (EuMC) and International Congress on Advanced Electromagnetic Materials in Microwaves and Optics (Metamaterials). Among his distinctions, Ferran Martín has received the 2006 Duran Farell Prize for Technological Research, he holds the Parc de Recerca UAB Santander Technology Transfer Chair, and he has been the recipient of two ICREA ACADEMIA Awards (calls 2008 and 2013). He is Fellow of the IEEE since 2012 and Fellow of the IET since 2016. 